\title{
Control of language use: cognitive modeling of the hemodynamics of Stroop task performance
}

\author{
Ardi Roelofs ${ }^{\mathrm{a}, \mathrm{b}, *}$, Peter Hagoort $\mathrm{t}^{\mathrm{a}, \mathrm{b}}$ \\ ${ }^{a}$ Max Planck Institute for Psycholinguistics, Wundtlaan 1, 6525 XD Nijmegen, The Netherlands \\ ${ }^{\mathrm{b}}$ F.C. Donders Centre for Cognitive Neuroimaging, Nijmegen, The Netherlands
}

8 September 2002

\begin{abstract}
The control of language use has in its simplest form perhaps been most intensively studied using the color-word Stroop task. The authors review chronometric and neuroimaging evidence on Stroop task performance to evaluate two prominent, implemented models of control in naming and reading: GRAIN and WEAVER ++ . Computer simulations are reported, which reveal that WEAVER ++ offers a more satisfactory account of the data than GRAIN. In particular, we report WEAVER ++ simulations of the BOLD response in anterior cingulate cortex during Stroop performance. Aspects of single-word production and perception in the Stroop task are discussed in relation to the wider problem of the control of language use.
\end{abstract}

(C) 2002 Elsevier Science B.V. All rights reserved.

Theme: Neural basis of behavior

Topic: Cognition

Keywords: Anterior cingulate; Executive control; Language; Latencies; Reading; Speaking; Stroop task

\section{Introduction}

The writer Oscar Wilde said that some people can resist everything except temptation. But if the ultimate goal of cognition is the adaptive control of perception and action, resisting temptation is the key to success. In achieving one's goals rather than being driven by distraction, executive or willed control plays a critical role, both in action generally and in verbal action specifically. For example, speakers need control to prevent interference from seeing irrelevant objects in the environment or hearing interlocutors while planning what they want to say. Bilinguals need control to prevent interference from one language when planning or processing the other. Executive control is not only needed to keep verbal perception and action focused in the face of distraction, but it is also required in monitoring for speech errors and their repair. Furthermore,

\footnotetext{
*Corresponding author. Tel.: +31-24-352-1347; fax: +31-24-3521213.

E-mail addresses: ardi@mpi.nl (A. Roelofs),
} peter.hagoort@fcdonders.kun.nl (P. Hagoort). the basic modes of language use-speaking, listening, reading, writing, and signing-seem to employ different configurations of basic processing components. When language users have to perform one particular verbal task rather than another, they are able to selectively control the configurations, usually without much practice. In some way or another, goals can be set adaptively to control language use and executive control helps to direct the machineries of perception and action to this goal.

Whereas the component processes serving speaking, listening, reading, writing, and signing have been domains of extensive empirical investigation (see Ref. [5] for reviews), the issue of their control has received much less attention. The willed control of verbal perception and action has in its simplest form perhaps been most intensively studied using the color-word Stroop task [43] and analogs of it. Following a long research tradition, we take the Stroop task to be a prototype of the willed control of language use. In Sections 2 and 3, we review some of the key chronometric and neuroimaging findings on Stroop task performance. In Section 4, we use the evidence to evaluate two prominent, implemented models of Stroop: 
GRAIN [4] and WEAVER ++ [22,39-41]. We report computer simulations showing that GRAIN provides a less satisfactory account of the data than WEAVER ++ . In Section 5, we place the findings on single-word production and perception during Stroop task performance in the broader context of the problem of the executive control of language use.

We start with a brief note on relevant distinctions. Willed or consciously wanted goals are often referred to as intentions. Not all goals in language use are willed. For example, the intention to verbally convey that the color of one's car is blue is presumably achieved through a hierarchy of subgoals including the goal of producing the sound /b/ by closing the lips. However, although lip closure may be a goal in verbal action, it is not a willed goal, although it may perhaps have been in the course of learning a language. That is, automatization through extensive practice may turn intentions into goals that are merely entailed by willed goals rather than being explicitly willed themselves. Intentions are illocutionary if they concern achieving communication through verbal means. Ultimately, language use is always driven by willed goals. Speakers, writers, and signers try to achieve communicative intentions through verbal means and listeners, readers, and viewers try to recover these intentions.

\section{Chronometric evidence on Stroop}

Performing a verbal task involves dynamically setting the language system to achieve a certain verbal goal. The Stroop task involves setting the goal to naming the ink color of color words (one task variant) or setting the goal to reading the words aloud (another task variant). That executive control is involved in Stroop task performance is evident from the finding that speakers are much slower and make more errors in naming the ink color of an incongruent color word (e.g. the word BLUE in red ink) than in naming the color of a colored series of Xs in the control condition or the ink color of a neutral word (e.g. the word DOG in red ink), referred to as Stroop interference. The ink colors are named rather than the color words read at the cost of slower responding and more errors. Furthermore, speakers are faster than neutral and control when color and color word agree in the congruent condition, referred to as Stroop facilitation. When the task is to read aloud the words and to ignore the ink colors, there is no interference from incongruent colors or facilitation from congruent colors relative to control, which is called the absence of a 'reverse Stroop' effect. Given that the words are read rather than the colors named, the absence of a reverse Stroop effect in reading shows that executive control in Stroop word reading is absent or involves no special temporal cost, unlike Stroop color naming.

The asymmetry in Stroop effects between color naming and word reading is not due to the relative speed of processing the colors and words. Color words are typically read some 100-200 $\mathrm{ms}$ faster than the corresponding colors are named [17]. However, when one compensates for the slower processing of colors by presenting color patches more than $200 \mathrm{~ms}$ in advance of the words to be read, still no interference on word reading is observed. Presenting the word before or after the color patch reduces interference in color naming. That is, interference peaks when color and word are presented simultaneously [17].

MacLeod [25] provides an extensive review of the Stroop literature, covering over 400 articles. Stroop interference not only occurs in naming colors while reading color words but it appears in many other verbal domains (e.g. position words interfere with the naming of positions, numerals interfere with the naming of numerosity, picture naming is interfered with by incongruent spoken words presented over headphones or written words superimposed on the picture). Stroop interference is also observed with typing the word. Consistent with the evidence for the dominant role of the left hemisphere of the brain in verbal processing, several visual half-field and evoked-potential (ERP) studies have observed greater Stroop interference in the left than in the right hemisphere. The magnitude of Stroop interference varies with age, with young children and older adults exhibiting the largest interference scores. Often, persons diagnosed with schizophrenia, frontal lobe lesions, and Alzheimer dementia show Stroop interference scores that are disproportionately high. Whereas monolinguals are not influenced by irrelevant words from a foreign language, Stroop interference does occur between the languages of bilinguals. The amount of interference is usually less between than within languages.

\section{Neuroimaging evidence on Stroop}

Neuroimaging studies have shown that an extensive network of brain areas is involved in Stroop task performance. Critical areas of this network are (1) the anterior cingulate cortex (ACC) and (2) the dorsolateral prefrontal cortex (DLPFC), both involved with executive control, (3) the left lingual gyrus subserving color processing and the left extrastriate cortex subserving visual word-form processing, and (4) the left perisylvian language areas including the areas of Broca and Wernicke. We briefly characterize the nature of the involvement of these areas in turn.

\subsection{Anterior cingulate cortex}

One of the brain areas that has quite consistently been associated with performing the Stroop task is the anterior cingulate cortex (ACC), which is located on the medial surface of the frontal lobes of the brain (see Ref. [32] for a review). Traditionally, the ACC is taken to be part of the 'limbic lobe', a term coined by Broca. Paus [32] characterized the ACC as the brain area where 'motor control, drive 
and cognition interface'. Firstly, extensive projections from the thalamus and brainstem nuclei to the ACC suggest an important role for drive and arousal in ACC involvement. Secondly, extensive reciprocal connections between the ACC and the DLPFC suggest an important role for cognition in ACC engagement. Thirdly, the motor areas of the ACC in the cingulate sulcus densely project to the spinal cord and the motor cortex, which suggests an important role for the ACC in motor control. The motor areas of the ACC contain subregions specifically controlling oculomotor, vocal, and manual responses.

In general, the ACC shows increased activation when performing a task is demanding and potentially error prone compared to when the task is easy (see Refs. [4,6,26] for reviews). The ACC shows increased activation in conflict compared to nonconflict conditions during response override tasks such as Stroop and the Eriksen flanker task. Furthermore, the ACC shows increased activation during tasks in which responding is underdetermined, such as in verb or noun generation from a verbal prompt compared to reading aloud the prompts. The ACC also shows increased activation in divided compared to selective perceptual attention tasks and during dual task performance compared to single task performance. Finally, the ACC is the locus of the error-related negativity (ERN, see Ref. [18] for a review), an evoked brain potential associated with awareness of error commission. More generally, the ACC seems to be involved in both behavioral and affective control functions [6,15,16,32].

As concerns Stroop color naming, brain imaging studies have shown that the ACC is more active in the incongruent than in the congruent condition [24,31,34]. Furthermore, neuroimaging studies indicate that both the incongruent and congruent conditions show more activation than the neutral condition [7] and the control condition [3]. Thus, interestingly, the pattern of ACC activations (incongruent $>$ congruent $>$ neutral/control) does not correspond to the chronometric ordering of the Stroop conditions (incongruent $>$ neutral/control $>$ congruent). Accounting for this dissociation between brain activations and color naming latencies constitutes a major challenge for models of Stroop [26].

An exception to the general pattern of ACC activations in Stroop is provided by the PET study of Taylor et al. [46]. Different from other studies, however, Taylor et al. gave their participants extensive practice on the Stroop task before scanning. Bench et al. [3] obtained only ACC activation in Stroop when the task was not practised in advance. This fits with previous observations that ACC activation decreases with practising a response [36].

The ACC is a large area with a complicated pattern of substructures [6,32]. Different subregions of the ACC appear to be involved with Stroop performance in different output modalities. Neuropsychological evidence for functional specialization within the human ACC comes from the Stroop performance of a patient (D.L.) with a focal lesion in the manual motor area of the ACC [47]. The patient was impaired on an arrow-word version of the Stroop task when giving manual responses but not when giving vocal responses. Lesion studies do not always provide a consistent picture of ACC involvement in Stroop, however. Extensive group studies of patients with ACC lesions have been reported, which did not provide evidence for impaired Stroop performance [44,48]. However, the patients differed in the locus and extent of damage within the ACC and also the time of onset and the cause of the lesion differed. The functional specificity of subregions of the ACC and the role of practice may perhaps account for some of the discrepancies among the neuropsychological results [45].

\subsection{Dorsolateral prefrontal cortex}

Another brain area that has been associated with aspects of executive control in performing the Stroop task is the dorsolateral prefrontal cortex (DLPFC). Activations of this area are typically observed in tasks that require maintenance and manipulation of information in working memory. Furthermore, the area is associated with goal or task manipulations (see Ref. [29] for a review). MacDonald et al. [24] provide evidence that the DLPFC serves to hold the task demands (goal) temporarily on-line in Stroop task performance. They observed differential activation of the DLPFC between color naming and word reading in task preparation before a Stroop trial, but no differential activation between the incongruent and congruent conditions in Stroop performance during the trial itself. In contrast, there was no differential activation of the ACC between color naming and word reading in task preparation before a Stroop trial, but the ACC was more active in the incongruent than in the congruent condition during a Stroop color naming trial itself.

\subsection{Lingual gyrus and extrastriate cortex}

Color perception is associated with the lingual gyrus (human V4) and various aspects of visual word-form processing with the left fusiform gyrus and other left and right extrastriate areas [12,36,37]. In a PET study of Stroop color naming, Carter et al. [7] observed a reduced blood flow to the left lingual gyrus in the congruent condition relative to the neutral condition and an increased blood flow in the incongruent condition relative to the congruent condition, the latter being in line with the activations of the ACC. The increased activation of the left lingual gyrus in the incongruent condition relative to the congruent condition has also been observed in other studies [31,35]. Furthermore, Carter et al. [7] observed a reduced blood flow to the left lateral extrastriate cortex in the incongruent Stroop condition compared to the congruent condition and in the congruent condition relative to the neutral condition. Thus, the pattern of activations of the left extrastriate area 
observed by Carter et al. is the reverse of the pattern of activations they observed for the ACC. Such negative correlations between ACC and visual cortex activations in Stroop have independently been observed by Bench et al. [3].

\subsection{Left perisylvian language areas}

In comparing brain activations during performance across the different Stroop conditions, activations of the core language areas are typically subtracted out. In their meta-analyses of 82 word production studies and 26 word perception studies, Indefrey and Levelt $[19,20]$ identified several core language areas for naming and reading. The analyzed studies included picture naming, word generation (e.g. generating a use for a noun, e.g. saying 'hit' to HAMMER), word reading, and pseudoword reading. As can be expected from the classic neuropsychology studies of Broca and Wernicke in the 19th century and most later studies, the neuroimaging studies localized the language areas basically in the left hemisphere. The middle part of the left middle temporal gyrus seems to be involved with conceptually driven lexical selection, which has been confirmed recently in an MEG study [27]. Next, activation spreads to Wernicke's area, where the phonological code of the word seems to be retrieved. Activation is then transmitted to Broca's area for post-lexical phonological processing such as syllabification. Finally, phonetic encoding and articulation take place, with contributions of the supplementary motor area (SMA), the cerebellum, and sensory-motor areas.

\section{Modeling Stroop task performance}

The discussions in the literature of executive control in the Stroop task have focused on the anterior cingulate cortex (ACC) and the dorsolateral prefrontal cortex (DLPFC). Whereas it seems generally accepted that the DLPFC plays a role in maintaining the task demands in working memory, there is much disagreement about the role of the ACC. Two major views of the involvement of the ACC in the Stroop task are the conflict monitoring view and the selection-for-action view. On the selectionfor-action view, the ACC plays a role in actually securing task-relevant control, that is, in implementing control $[1,18,26,36]$. In contrast, the conflict monitoring view $[7,24]$ holds that the ACC merely signals the presence of conflict or crosstalk between processing channels, prompting other systems to actually implement the control. The conflict monitoring view has recently been computationally implemented in the GRAIN model of Stroop task performance [4].

In Sections 4.1. and 4.2, we argue that GRAIN needs to be modified to account for critical aspects of the data discussed in Sections 2 and 3, in particular, the findings on
ACC activation and the activations of the posterior visual areas. In Sections 4.3 and 4.4 , we explain the selection-foraction view as recently implemented in the WEAVER ++ model of spoken word production [22,39-41]. We report simulations showing that this model not only accounts for the Stroop latency findings but also for the ACC activations. Furthermore, we propose an extension of the model to account for the activations of the DLPFC and the visual areas.

\subsection{The GRAIN model of Stroop performance}

The GRAIN (Graded Random Activation-based Interactive Nonlinear) model of performance in the Stroop task developed by Cohen and Huston [9] assumes that the task is achieved through an interactive-activation network consisting of input, output, and task demand nodes, which is illustrated in Fig. 1. Input nodes represent perceived words and colors. Processing occurs through activation spreading from input to output nodes, whereby nodes change their activation with time in a continuous, nonlinear manner. There are between-level excitatory links and within-level inhibitory links. The connections between word input nodes and response output nodes in the word reading pathway are stronger than the connections between color input nodes and the same response nodes in the color naming pathway, reflecting a greater practice in reading than naming. Following Norman and Shallice [30] and others, the executive control of color naming and word reading is achieved by biasing the activation levels of responses. In particular, color naming and word reading are achieved by activating the corresponding task demand node. On each simulated Stroop trial, task input is given and activation cycles around until a stable pattern of activation (an 'attractor') is reached. Next, the relevant color and word input nodes are activated. The output node

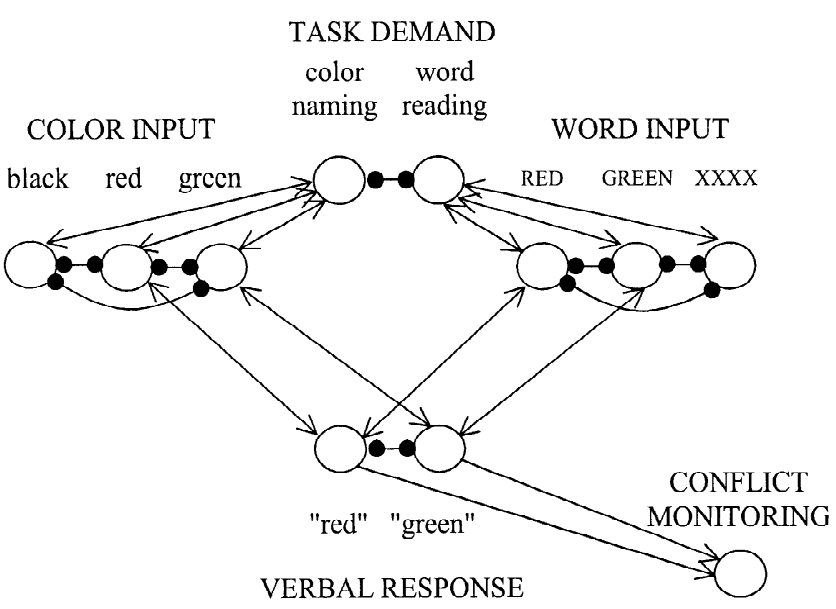

Fig. 1. Network of the GRAIN model [9] and its extension with conflict monitoring by Botvinick et al. [4]. $\longrightarrow$ denotes a bidirectional inhibitory connection and $\leftrightarrow$ denotes a bidirectional excitatory connection. 
whose activation first exceeds a threshold is chosen as response.

The GRAIN model successfully accounts for the Stroop interference in color naming and for the absence of interference in word reading [9]. In color naming, a distractor word puts the response layer in an attractor for the perceived word rather than the color. The Stroop interference is explained as the extra time it takes to move from the attractor for the word into the attractor for the color name. There is no interference in word reading because the weaker color naming pathway is much less effective in putting the system in the wrong attractor. The model is not successful in accounting for the time course of Stroop interference, however. Simulations by Cohen and Huston [9] showed that interference in the model does not vary with the pre-exposure time of the distractor, whereas in the real data, interference decreases when the preexposure time increases [17]. After receiving word input, the GRAIN network quickly settles into a stable state of activation for the response corresponding to the word. The stable state does not vary with time, hence making the pre-exposure time of the distractor longer or shorter has no effect, until a pre-exposure time is used that is too short for the distractor to reach an attractor. Consequently, the amount of time it takes for the color name to overcome the interference from the word is constant, in disagreement with the real data (see Ref. [41] for discussion).

\subsection{Simulation of Stroop performance by GRAIN}

Recently, Botvinick et al. [4] implemented the conflict monitoring view of the ACC in GRAIN by adding a 'conflict-monitoring' node to the model's network. The conflict-monitoring node has an 'energy' reflecting the degree of conflict in the response layer, with $a_{i}$ and $a_{j}$ being the activation levels of nodes $i$ and $j$ and $w_{i j}$ the strength of the connection between them, whereby $\operatorname{energy}(t)=-\sum_{i} \sum_{j} a_{i}(t) a_{j}(t) w_{i j}$.

The conflict monitoring node stands for the contribution of the ACC and the task demand nodes for the contribution of the DLPFC. The extended GRAIN model was applied to both latency and neuroimaging data on Stroop task performance. Simulations by Botvinick et al. [4] showed that the conflict monitoring node was more active in the incongruent than in the congruent condition and that both conditions were more active than the control condition, in line with the empirically observed ACC activations [3,7]. Furthermore, responding in the incongruent condition was slower than in the control condition and slower in the control condition than in the congruent condition, as empirically observed [3,7]. The left-most panel of Fig. 2 shows the energy of the response layer as a function of Stroop condition.

The empirical neuroimaging study of Carter et al. [7] included the neutral condition (the word DOG in a color) rather than the control condition (a row of Xs in color) used in the simulations by Botvinick et al. [4]. When we ran simulations of the GRAIN model with DOG rather than the row of Xs, the response-layer energy was virtually the same with the neutral word DOG and the congruent color word, contrary to the neuroimaging data [7]. Furthermore, the color naming latencies were the same with neutral words and incongruent words, in disagreement with the empirical data [3,7]. It has been argued that words that are not part of the response set (like DOG) receive a negative bias in the response layer [8]. Implementing such a bias (and testing a wide range of bias values) resulted in slightly greater response-layer energies in the congruent than in the neural condition, as empirically observed. However, the color naming latencies in the neutral and incongruent conditions remained the same, contrary to the empirical data.

We also found other discrepancies between model and data. Our simulations revealed that GRAIN predicts that

\section{GRAIN}

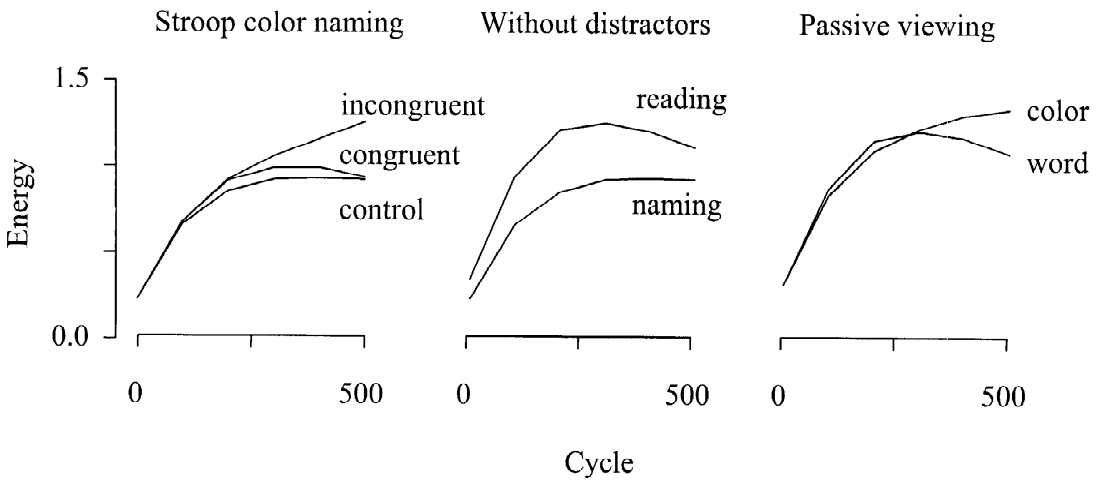

Fig. 2. Characteristic conflict node energies in the GRAIN model in the incongruent, congruent, and control conditions during performing the Stroop color naming task, during bare color naming and word reading, and during passive color and word perception. 
the ACC should be active to a similar extent when the task is Stroop color-word naming and when no word distractor is presented, that is, when the task is bare color naming. The simulations of bare color naming were run by activating the color-naming task node and one of the color input nodes (e.g. red), whereas a word input node was not activated. The middle panel of Fig. 2 shows the amount of energy in the response layer during bare color naming. The energy curve shown is typical, although the exact energy values depend on the parameter values in the model. Due to the interactive nature of processing in the model (all nodes are bidirectionally connected) and the activation of multiple input nodes by a task node, activating a single color input node leads to the activation of multiple responses in GRAIN. Consequently, the energy level in the model is high even without conflicting stimulus information. For the same reasons, GRAIN predicts that the ACC should be active to a similar extent when the task is Stroop color-word naming and when the task is bare word reading, which is also shown in the middle panel of Fig. 2. The energy of the response layer is higher with bare word reading than with bare color naming, because the connections between the word input nodes and the response nodes are stronger than the connections between the color input nodes and the response nodes. Therefore, the response nodes are more active with bare word reading than with bare color naming, and the energy of the response layer is higher with reading than with naming. Thus, GRAIN predicts more ACC activation when the response is highly practised (e.g. reading aloud the word RED) than when it is not (e.g. naming the ink color red).

Moreover, GRAIN predicts that the ACC should be active to a similar extent with Stroop color-word naming and with passive color or word perception, that is, when no task input and no distractor input is given, as the rightmost panel of Fig. 2 shows. Our simulations of passive color and word perception were run by activating one of the color input nodes (for color perception) or one of the word input nodes (for word perception), whereas no task demand node was activated. Again, due to the interactive nature of processing in the model, activating a single color or word input node and no task node still leads to the activation of multiple responses in GRAIN. As a result, the energy level in the model is high, even without conflicting stimulus information. Running the model with a low-level control condition such as 'keeping the eyes closed' (i.e. providing no input at all to the network and inhibiting the response layer) yielded not much activation of the conflict monitoring node. Thus, GRAIN predicts that passive viewing should yield high ACC activation compared to keeping the eyes closed, but Stroop color naming and passive viewing should yield comparable ACC activations.

None of the above predictions of the model is supported by the empirical data, however. Empirically, more ACC activation is observed in conflict compared to non-conflict situations [4,6,26,36]. The ACC is not differentially activated with passive color perception and passive word perception compared to keeping the eyes closed [19,20,36]. Moreover, ACC activation decreases rather than increases with practising a response $[36,46]$.

Furthermore, the neuroimaging evidence on Stroop color naming suggests that the lingual gyrus subserving color processing is more active in the incongruent condition than in the congruent condition and more active in the neutral than in the congruent condition [7,31,35]. The left panel of Fig. 3 shows the activation of the color input layer of GRAIN over time as a function of Stroop condition. As can be seen, the activations of the color layer in GRAIN are virtually the same in the different Stroop conditions, in disagreement with the neuroimaging data [7,31,35]. Moreover, the neuroimaging evidence on Stroop color naming suggests that the extrastriate cortex subserving visual word-form processing is more active in the neutral than in the congruent condition and more active in the congruent condition than in the incongruent condition [7]. The right panel of Fig. 3 displays the activation of the word input

GRAIN

Color input nodes

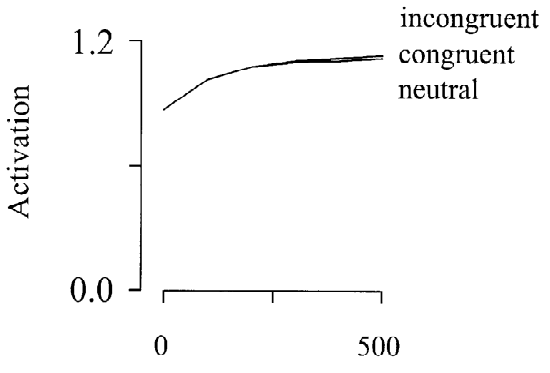

Cycle
Word input nodes

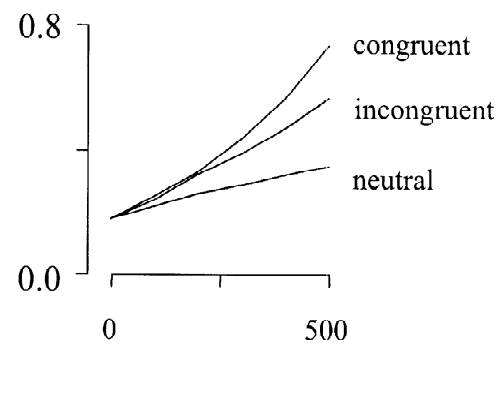

Fig. 3. Characteristic color and word input node activations in the GRAIN model in the incongruent, congruent, and neutral conditions during performing the Stroop color naming task. The plotted activations are the sums of the activations of the nodes in the color and word input layers. 
layer over time as a function of Stroop condition in GRAIN. The figure shows that the word input layer in GRAIN is least active in the neutral condition, in disagreement with the empirical findings.

In summary, the GRAIN model accounts for some of the basic chronometric findings on Stroop, although it fails to account for the time course of the Stroop phenomenon. Furthermore, whereas the conflict monitoring view implemented in GRAIN accounts for the ACC activations and response times in some of the conditions of the color-word Stroop task, the model fails to account for the absence of ACC activation in passive color and word perception compared to keeping the eyes closed. Furthermore, the model fails to account for the finding that ACC activation is reduced when the response is highly practised compared to when it is not. Finally, the pattern of activations of color and word input nodes in the model disagrees with the pattern of activations of the cortical areas subserving color and visual word-form processing.

It should be noted, however, that in order to derive the predictions from GRAIN, we ran the model in situations that the original developers of GRAIN had not yet modeled (e.g. the neutral Stroop condition, color naming without distractor, passive viewing). In applying the model to these novel situations, we had to make new assumptions. For example, we linked activations of the color and word input nodes in GRAIN to activations of the color and word form areas of the brain. The developers of GRAIN might have adopted somewhat different or more complex assumptions about the best way to model the task variants and might have had more success. Thus, the predictive failures of specific instantiations of the GRAIN architecture need not imply that the architecture itself is flawed. The data challenge rather than refute GRAIN and point to the need of further developing the model.

\subsection{The WEAVER ++ model of Stroop performance}

Recently, a new account of the color-word Stroop phenomenon has been proposed based on an implemented model of spoken word production, WEAVER $++[22,39-$ 41]. On this account, Stroop effects arise from processing interactions among representations within the languageproduction architecture and task-relevant control achieved through explicit reference to goals, following Anderson [2], Levelt [21], and others. It has been shown that WEAVER + + successfully simulates 16 classic data sets on Stroop, mostly taken from the review by MacLeod [25], including incongruency, congruency, reverse Stroop, response set, semantic gradient, time course, stimulus, spatial, multiple task, manual, bilingual, training, age, and pathological effects (see Ref. [41]). With only three free parameters taking two values each to accommodate task differences (color naming, picture naming, word reading, manual responding), the model accounts for $96 \%$ of the variance of the 16 studies (250 data points).
WEAVER ++ implements the view that naming a perceptual entity such as a color involves a hierarchy of processing stages, illustrated in Fig. 4. Firstly, there is the conceptual identification of the color based on perceptual input (e.g. red) and its designation as goal concept (i.e. $\operatorname{RED}(\mathrm{X})$ ). Secondly, the lemma of the corresponding word is retrieved (i.e. $r e d$ ), in the Stroop literature often referred to as response selection (except that it involves here lemmas). A lemma is a representation of the syntactic properties of a word, crucial for its use in sentences [22]. Thirdly, the form of the word is encoded (i.e. [red]), called response programming. Finally, the name is articulated, called response execution. A perceived written word activates its lemma and its output form in parallel. Oral reading is achieved by a shallow form-to-form route (e.g. from the orthographic form RED to [red]) or may involve an extra step of lemma retrieval (i.e. from RED via red to [red]), roughly corresponding to what is traditionally called the 'semantic' route in reading aloud.

The model assumes that the mental lexicon is a network with information about words, a small fragment of which is illustrated in Fig. 5. A conceptual stratum represents concepts as nodes in a semantic network. For example, the concept red is represented by the node $\operatorname{RED}(\mathrm{X})$. The syntactic stratum contains lemma nodes, such as red, which are connected to nodes for their syntactic class (e.g. adjective). And finally, the form stratum contains nodes representing morphemes (e.g. $\langle$ red $\rangle$ ), segments (e.g. /r/),

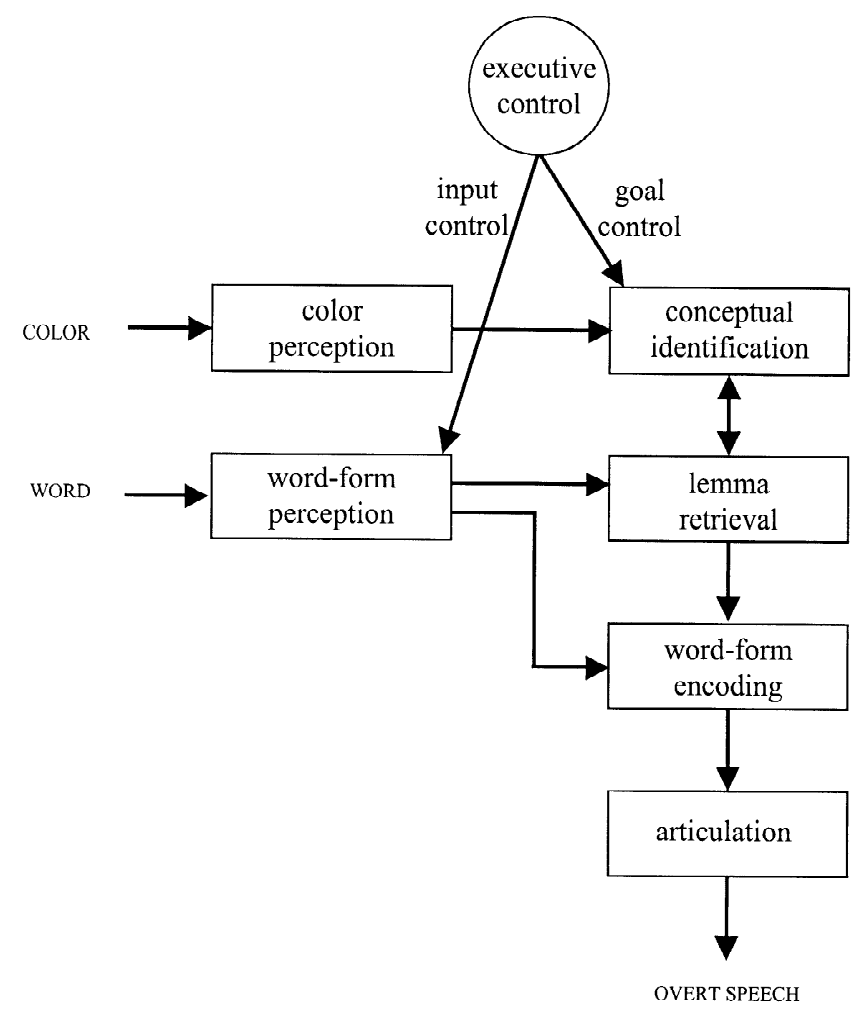

Fig. 4. Flow of information and control in the WEAVER ++ model $[22,41]$. 


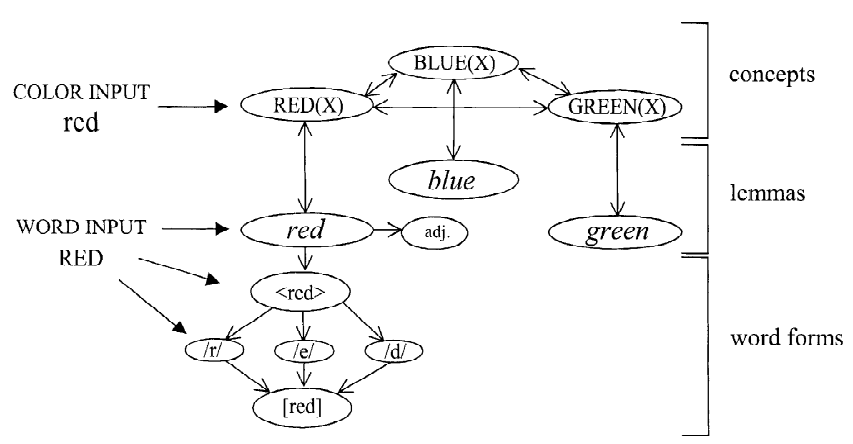

Fig. 5. Network of the WEAVER ++ model. Only a small fragment of the network is shown. $\rightarrow$ denotes a unidirectional excitatory connection and $\leftrightarrow$ denotes a bidirectional excitatory connection.

and motor programs (e.g. [red]). Information is retrieved from the network by spreading activation. For example, a perceived color (e.g. red) activates the corresponding concept node (i.e. $\operatorname{RED}(X)$ ) in the network. Activation then spreads through the network following a linear activation rule with a decay factor. Each node sends a proportion of its activation to the nodes it is connected to. For example, $\operatorname{RED}(X)$ sends activation to other concepts such as BLUE(X) and also to its lemma node red. Selection of nodes is accomplished by production rules specifying condition-action pairs [2]. A rule is triggered when its nodes become active. A lemma retrieval production rule selects a lemma if the connected concept is the goal concept. For example, red is selected for $\operatorname{RED}(\mathrm{X})$ if it is the goal concept and red has reached a critical difference in activation compared to other lemmas. Thus, how fast a node is selected depends on how active the other nodes are.

According to the selection-for-action view implemented in WEAVER ++ , the ACC plays a role in implementing task-relevant control [41]. According to the model, ACC activation reflects executive processing, whereas the Stroop conflict arises during selections in planning the target word. The latter happens in the left perisylvian cortex, including the areas of Wernicke and Broca (see Refs. $[19,20]$ for details). Executive control in the model includes goal control and input control. Goal control consists of ensuring that response selection confirms the task demands and that inappropriate responses are suppressed. Input control consists of suppressing input from the irrelevant perceptual channel, for example the word channel in color naming.

If ACC activation reflects word reading suppression, the area should be more active in the incongruent than in the congruent condition and both conditions should be more active than the neutral condition, as empirically observed [7]. Suppressing the reading response in the incongruent and congruent conditions depends on color name selection: incongruent and congruent distractors are also color words and therefore possible responses. Suppressing the selection of the lemma of a read color word can only occur if the read word is not the name of the color, which is only evident after the name of the color is selected (which takes longer in the incongruent than in the congruent condition). However, if a neutral word is read, suppressing the reading response does not have to wait till the color name is selected. Consequently, if ACC activation reflects reading response suppression, the incongruent condition should be more active than the congruent condition and both conditions should be more active than the neutral condition, as empirically observed [7].

In contrast, the differences in color naming latencies among the Stroop conditions reflect differences in the color name selection times [41]. In WEAVER ++ , lemma selection times are longer in the incongruent condition than in the neutral condition and they are longer in the neutral condition than in the congruent condition, as empirically observed [7,25]. Thus, whereas the reading of neutral words can be quickly suppressed (reflected in the ACC activation), the selection of the color name takes longer with neutral than with congruent words (reflected in the color naming latencies). This is because selection of the color name is primed by congruent words but not by neutral words.

The meta-analyses of neuroimaging studies on naming and reading by Indefrey and Levelt $[19,20]$ suggest that conceptually driven lemma retrieval is associated with the middle part of the left middle temporal gyrus, confirmed in an MEG study [27]. However, this area is typically not differentially activated among the Stroop conditions in PET and fMRI studies of Stroop performance, although the mid-temporal area has been found to be more active in the incongruent than the congruent Stroop condition in one fMRI study [35]. A reason for this discrepancy among studies may be the different sensitivity of the various neuroimaging techniques to processes in the brain. The temporal resolution of MEG is much higher than that of PET and fMRI. Furthermore, whereas MEG measures neural activity directly, PET and fMRI measure it only indirectly through the hemodynamic response to neural activity. Whether a differential hemodynamic response is obtained critically depends on the amount and duration of neural activity and the brain areas involved. Thus, it remains possible that MEG is able to register the activation associated with lemma competition while the activation is not so readily registered with PET and fMRI. An MEG study of Stroop would be welcome.

\subsection{Simulation of Stroop performance by WEAVER ++}

We tested the Stroop account of WEAVER ++ given above by running computer simulations. As target data for the simulations, the fMRI study of MacDonald et al. [24] and the PET study of Carter et al. [7] were taken. The fMRI study measured the hemodynamic response in the ACC during the incongruent and congruent Stroop conditions and the study also measured the corresponding 


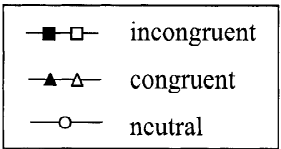

fMRI observed (ACC)

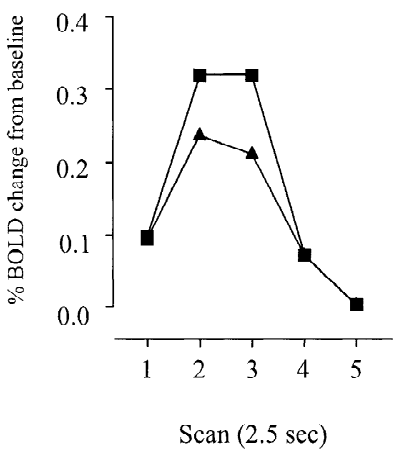

WEAVER++

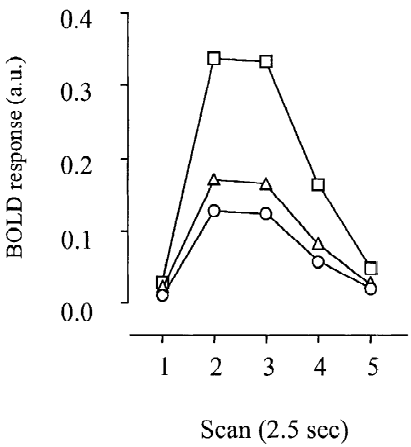

Fig. 6. BOLD responses in the Stroop conditions as empirically observed by MacDonald et al. [24] and in WEAVER ++ simulations $(\mathrm{du}=100 \mathrm{~ms}$, critdiff $=1.2$ ). The difference in speech production latency between the incongruent and congruent conditions was $116 \mathrm{~ms}$ in the fMRI study and $127 \mathrm{~ms}$ in the simulations.

color naming latencies. The PET study measured the ACC activations during the incongruent, congruent, and neutral Stroop conditions as well as the corresponding color naming latencies.

The simulations were run as follows. For each time step $\Delta t$ during which goal control and input control was required, a node representing the contribution of the ACC received input with a size equal to the standard input parameter of the model. The sum of the inputs, referred to as $w$, is an index of the amount of control needed in the different Stroop conditions. It was assumed that a gamma function provides a reasonable model of the BOLD response [10]. For the simulations it was assumed that

BOLD response $(t)=w t^{\mathrm{du} / \Delta t} e^{-t / \text { critdiff }}$

where du and critdiff are parameters of the model. The du (duration) parameter is the latency of input control and critdiff (critical difference) is the response threshold in the model. The response latencies were derived as in earlier simulations of the model [39-41].

The left panel of Fig. 6 shows the BOLD response in the ACC on incongruent and congruent trials measured by MacDonald et al. [24]. The right panel shows the corresponding BOLD responses in WEAVER ++ simulations. The difference in color naming times between the incongruent and congruent conditions measured by MacDonald et al. was $116 \mathrm{~ms}$ and it was $127 \mathrm{~ms}$ in the WEAVER + + simulations. Thus, the larger hemodynamic response and the larger color naming latency in the incongruent than in the congruent condition observed by MacDonald et al. are also observed in the WEAVER ++ simulations. Furthermore, the simulations yielded a larger hemodynamic response in the incongruent and congruent conditions than in the neutral condition, which corresponds to what Carter et al. [7] observed in their PET study. The left panel of Fig. 7 shows the corresponding color naming latencies observed by Carter et al. [7] and the right panel shows the latencies in WEAVER ++ simulations. To conclude, WEAVER ++ agrees with the empirical data.

Although WEAVER ++ does not include detailed assumptions about goal maintenance, color perception, and
RT observed

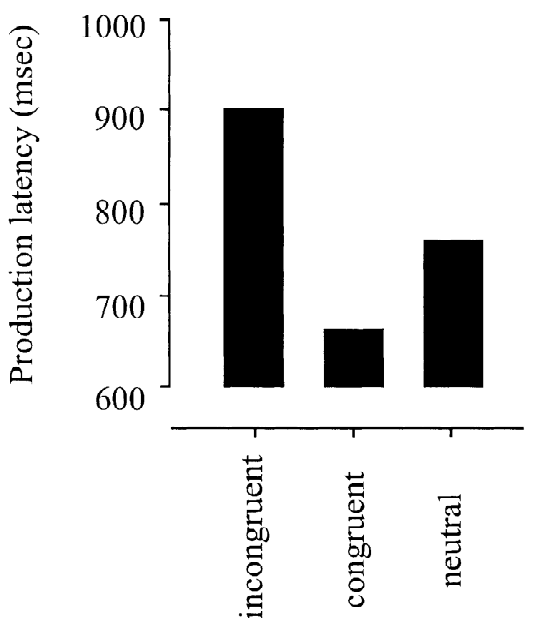

WEAVER++

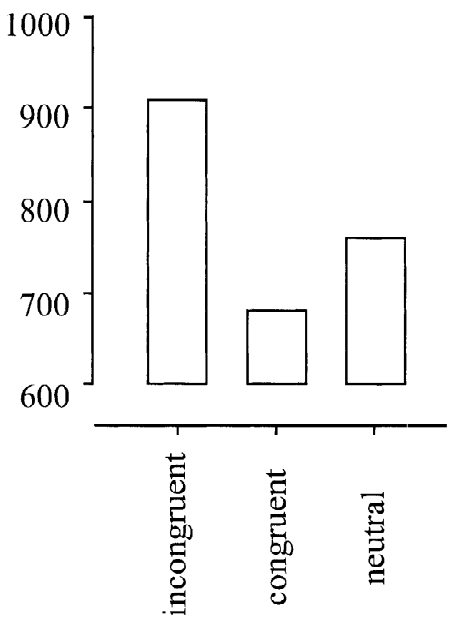

Condition

Fig. 7. Stroop color naming latencies as empirically observed in the PET study by Carter et al. [7] and in WEAVER ++ simulations (du $=200 \mathrm{~ms}$, critdiff $=1.2$ ). The absolute color naming latencies were obtained in the simulations by adding a constant of 605 ms to the simulated response selection latencies. For the ACC, activations measured by Carter et al. revealed that incongruent $>$ congruent $>$ neutral. 
visual word-form perception, the model is compatible with the pattern of activations of the DLPFC and the color and visual word-form processing areas of the brain. If the DLPFC is the brain structure responsible for maintenance, one expects the area to be less active with a highly practised task (reading) than with a less practised task (color naming), as empirically observed [24]. If perceived colors are attended to until the goal (color naming) is reached [41], the ordering of activations of color input representations should follow the ordering of the color naming latencies in the Stroop conditions, which corresponds to what is empirically observed $[7,31,35]$. Furthermore, if the activation of word-form input representations reflects input suppression, then the ordering of activations of these representations should be the reverse of the ordering of ACC activations in the different Stroop conditions, which corresponds to what is empirically observed [7]. As it stands, WEAVER ++ has a less detailed story to tell about goal maintenance and the DLPFC than GRAIN. Clearly, further development of WEAVER ++ is warranted.

\section{Discussion and conclusions}

We have reviewed chronometric and neuroimaging evidence on Stroop task performance to evaluate two implemented models of executive control in language: GRAIN and WEAVER ++ . GRAIN implements the view, prominently advocated by Norman and Shallice [30], that executive control is achieved by associatively biasing activation levels of perceptions and actions. Goals are sources of activation that predispose for certain actions, but the selection of actions does not make explicit reference to these goals. WEAVER ++ implements the view, prominently advocated by Anderson [2], that actions are controlled through explicit reference to goals. Goal-referenced control assures that actions and perceptions remain focused and it ensures that decisions can be made between actions. It appeared that WEAVER ++ offers a more satisfactory account of the empirical data on Stroop than does GRAIN.

Although single word production and perception make up an essential ingredient of language use, the principles underlying the control of single words do not necessarily hold for the production and comprehension of sentences and discourses. In this final section, we relate aspects of Stroop task performance to the wider problem of the control of language production and perception. We argue that the principles underlying the control of single words and discourses are essentially the same. Furthermore, we briefly address the control of multiple languages, the role of learning and practice, and the relation between conflict monitoring and self-monitoring.

\subsection{From single words to discourses}

The use of language in conversational speaking, listening, reading, writing, and signing does not occur in isolation, but it is typically embedded in other activities. Speakers may plan their turn in a conversation while listening to their interlocutor and while walking or driving a car. They may watch an event or scene while listening to an interlocutor and they may listen to the radio or to music while reading the newspaper or writing a letter. Furthermore, speakers not only listen to their interlocutors while planning their turn in a conversation, but they also monitor their own internal and overt speech for errors and appropriateness [21].

In both written and spoken sentence comprehension, words come in one by one. Similarly, in sentence production, words are produced one after the other. It has therefore been argued, independently for comprehension and production, that processing and planning proceed in an incremental fashion rather than in parallel. As they come in, perceived words trigger procedures that compute their syntactic relations and meanings in the ongoing discourse, and words planned to express meanings within a discourse trigger procedures that compute their syntactic relations and prosodic forms [5,21].

The communicative intentions in discourses are typically much more complex than those in a laboratory task such as Stroop. For example, rather than the intention to name ink colors, a speaker may have the intention to convey that the color of his car is blue or that he saw that the color of the car of his boss is white. This points to the need for a combinatorial system such as a production rule system that can incrementally represent an infinitive number of intentions rather than a few fixed ones. Furthermore, it asks for a combinatorial system that can incrementally express those intentions verbally. The principles of control in word planning and perception may be the same for single and multiple word utterances, however. For example, if $\operatorname{BLUE}(\mathrm{X})$ is made the goal concept and lemmas are selected in a goal-referenced fashion, the lemma of blue will be selected, regardless of whether the concept $\operatorname{BLUE}(\mathrm{X})$ is part of the intention to name ink colors or to produce a sentence stating what the color of one's car is. Similarly, goal-referenced selection will protect the planning of blue from interference regardless of the complexity of the intention. To conclude, although conversations are driven by time-varying structured intentions, the principles of their control may be the same as for single words.

\subsection{Control of multiple languages}

Bilinguals need executive control to protect the use of one language against the inadvertent use of the other language. Color naming in a bilingual Stroop experiment 
not only asks for naming the colors rather than reading the words, but it also asks for responding in the appropriate language. This may be achieved by including language nodes and language-specific response nodes in GRAIN or by specifying the target language in the production rules and lemmas in WEAVER ++ .

Stroop interference between languages is about $60-70 \%$ of that within a language (see Ref. [14] for a review), which has been simulated successfully by WEAVER ++ [41]. Stroop interference between languages suggests that words of the non-target language are processed up to the conceptual level. This assumption agrees with the results of a wide variety of chronometric studies of bilingual word perception (see Ref. [13] for a review), but it is at odds with the conclusion from a recent neurophysiological and fMRI study by Rodriguez-Fornells et al. [38]. In this study, bilingual Spanish/Catalan and monolingual Spanish participants were instructed to press a button when presented with words in Spanish and to ignore pseudowords and words in Catalan. The evoked brain potentials (i.e. the $\mathrm{N} 400$ ) of both the bilinguals and monolinguals were sensitive to the frequency of the words in Spanish but not in Catalan. Furthermore, the fMRI response to Spanish words revealed greater activation of the posterior inferior frontal cortex and the planum temporale for the bilinguals than for the monolinguals. Previous research has suggested that these areas are involved in phonological processing [19]. According to Rodriguez-Fornells et al., these results suggest that bilinguals can selectively shut down one of their lexicons. In particular, bilinguals prevent interference from the non-target language by accessing the lexicon of the target language through application of language-specific sublexical grapheme-to-phoneme correspondence rules and blocking direct lexical access for both languages.

This account of the selective processing of languages in bilinguals assumes that language users can control whether a lexical or sublexical route into the lexicon is taken in reading and that they have control over the type of processing within the sublexical route. That is, the account assumes that bilingual readers can control which set of grapheme-to-phoneme correspondence rules is applied (i.e. the rules of Spanish or Catalan). However, there exists no good evidence for the executive control over lexical versus sublexical reading routes and over grapheme-to-phoneme correspondence rules [23]. Moreover, if readers can block the direct route into the mental lexicon and can control which set of grapheme-to-phoneme correspondence rules is applied, the findings on bilingual Stroop interference remain unexplained. If routes and processes within routes can be controlled, the simplest way to prevent betweenlanguage interference in color naming would be to block both the direct lexical route and the application of grapheme-to-phoneme correspondence rules for the nontarget language or to block all reading routes for all languages. The findings on between-language Stroop inter- ference suggest that bilinguals are unable to achieve such blockings.

The absence of an effect of non-target language words on brain potentials and a difference in activation of brain areas between monolinguals and bilinguals in fMRI does not necessarily imply, however, that bilinguals are able to selectively shut down one of their lexicons, as RodriguezFornells et al. [38] maintain. The bilingual Stroop results indicate that between-language effects on responding are attenuated compared to within-language effects. Attenuated effects for words from the non-target language also may lead to an absence of an effect on brain potentials. Furthermore, the availability of two languages rather than one language may lead to more extensive word processing before responding in bilinguals compared to monolinguals, which may explain why certain brain areas are more active in bilinguals than in monolinguals.

\subsection{Role of learning and practice}

Intentional or willful behaviors are typically learned and so depend on a system that can find out, represent, and store what resources are required to achieve the goals. The ACC and DLPFC seem to provide such a system (see Refs. $[18,29]$ for reviews and discussions). Stroop interference has often been interpreted in terms of a greater strength of processing or automaticity of reading compared to color naming, a view which has been implemented in GRAIN by assuming stronger connections in the reading than in the color naming pathway. On this account, words interfere with color naming because reading is a more practised and hence a more automatic or stronger process than color naming. The more automatic a process, the more it is capable of interfering with a less automatic process. In adults, reading is assumed to be very automatic and color naming much less so. Hence words interfere with color naming but colors not with reading. This view predicts that the Stroop conflict should increase as reading becomes more practised in children in the course of learning to read. However, the empirical findings show exactly the opposite pattern. The Stroop conflict for color naming decreases with increased skill in reading of children [42]. The magnitude of Stroop interference varies with age, with the youngest children and the older adults exhibiting the largest interference scores [11].

By assuming a strengthening and weakening with age of control structures rather than of processing pathways, WEAVER ++ has been able to simulate successfully the pattern of Stroop performance across the life span [41]. Independent evidence for this assumption comes from developmental studies (see Ref. [6] for a review). There is an extensive development of the connectivity of the ACC from infancy to late childhood. The size of the ACC of children correlates with response times in controlled but not in simple tasks. Furthermore, a substantial develop- 
ment of the general ability to control conflict has been found to occur between 2 and 5 years of age [6].

\subsection{Conflict monitoring and self-monitoring}

During conversation, speakers not only talk and listen to interlocutors, but they also monitor their own covert and overt speech for errors. Similarly, in performing a Stroop task, speakers monitor their performance (in Stroop's original experiments [43], participants had to repair their errors). To account for this type of output control, models need to make assumptions about self-monitoring and repair. Self-monitoring is more than conflict monitoring. GRAIN implements the view that the presence of crosstalk interference between processing channels triggers the system to increase the activation level of the relevant task demand node [4]. However, verbal self-monitoring involves more than this because it provides information about the nature of the trouble or failure, for example, whether a lexical error (e.g. 'blue' for 'red') or phoneme error (e.g. 'bed' for 'red') was made. This information drives covert or overt repairs [21].

Self-monitoring may be achieved through the speech comprehension system and may involve both evaluating the speech plan before it is articulated as well as listening to self-produced overt speech $[21,22]$. That not only tells whether the planning was successful in terms of the ultimate goal (e.g. whether the ink color was named rather than the word read) but it also tells about the nature of the occasional trouble or failure. This information allows for a corrective action in terms of an overt or covert repair (e.g. selecting / $/ \mathrm{r} /$ instead of $/ \mathrm{b} /$ if bed rather than red had been planned) or in terms of increasing the goal activation $[2,21]$. The view of self-monitoring through the speech comprehension system agrees with the results from neuroimaging studies, which suggest that self-monitoring and speech comprehension are served by the same neural structures [28,33]. Awareness of making an error yields the ERN [18].

Awareness of the outside world (perception), control of action (will), and awareness of one's own mental states and those of others are critical aspects of consciousness. Language use is impossible without all three aspects. Language production aims at conveying a communicative intention and language comprehension aims at recovering the communicative intention. Without the capacity to represent the intentions of oneself and those of others, communication cannot happen. Similarly, without the capacity to perceive, articulate, write, or sign, verbal communication cannot occur. But representing intentions and perceiving and producing linguistic signs is not sufficient. Without the capacity to keep to one's own goals, language users would only be reacting to what they hear or see rather than following their own communicative agenda. One may speculate that awareness of activity in the system that secures one's own goals gives rise to the feeling of 'being in control', that is, exercising one's will-or the feeling that the outcome was not completely satisfactory, resulting in the ERN [18]. Thus conceived, activations of the ACC and DLPFC constitute the neural correlate of the Stroop task demonstration that most language users can resist anything, even temptation.

\section{Acknowledgements}

We are indebted to Miranda van Turennout, Michael Coles, Pim Levelt, Peter Indefrey, Rasha Abdel-Rahman, and an anonymous reviewer for helpful comments and discussions.

\section{References}

[1] A. Allport, Attention and control: have we been asking the wrong questions? A critical review of 25 years, in: D. Meyer, S. Kornblum (Eds.), Attention and Performance XIV: A Silver Jubilee, MIT Press, Cambridge, MA, 1993, pp. 183-218.

[2] J.R. Anderson, The Architecture of Cognition, Harvard University Press, Cambridge, MA, 1983.

[3] C.J. Bench, C.D. Frith, P.M. Grasby, K.J. Friston, E. Paulesu, R.S.J. Frackowiak, R.J. Dolan, Investigations of the functional anatomy of attention using the Stroop test, Neuropsychologia 31 (1993) 907922.

[4] M.M. Botvinick, T.S. Braver, D.M. Barch, C.S. Carter, J.D. Cohen, Conflict monitoring and cognitive control, Psychol. Rev. 108 (2001) 624-652.

[5] C.M. Brown, P. Hagoort (Eds.), The Neurocognition of Language, Oxford University Press, Oxford, 1999.

[6] G. Bush, P. Luu, M.I. Posner, Cognitive and emotional influences in anterior cingulate cortex, Trends Cogn. Sci. 4 (2000) 215-222.

[7] C.S. Carter, M. Mintun, J.D. Cohen, Interference and facilitation effects during selective attention: an $\mathrm{H}_{2}^{15} \mathrm{O}$ PET study of Stroop task performance, NeuroImage 2 (1995) 264-272.

[8] J.D. Cohen, K. Dunbar, J. McClelland, On the control of automatic processes: a parallel distributed processing account of the Stroop effect, Psychol. Rev. 97 (1990) 332-361.

[9] J.D. Cohen, T.A. Huston, Progress in the use of interactive models for understanding attention and performance, in: C. Umiltà, M. Moscovitch (Eds.), Attention and Performance XV: Conscious and Nonconscious Information Processing, MIT Press, Cambridge, MA, 1994, pp. 453-476.

[10] M.S. Cohen, Parametric analysis of fMRI data using linear systems methods, NeuroImage 6 (1997) 93-103.

[11] P.E. Comalli, S. Wapner, H. Werner, Interference effects of Stroop color-word test in childhood, adulthood, and aging, J. Genet. Psychol. 100 (1962) 47-53.

[12] S. Dehaene, L. Naccache, L. Cohen, D. Le Bihan, J.-F. Mangin, J.-B. Poline, D. Rivière, Cerebral mechanisms of word masking and unconscious repetition priming, Nat. Neurosci. 4 (2001) 752-758.

[13] T. Dijkstra, W.J.B. Van Heuven, The architecture of the bilingual word recognition system: from identification to decision, Bilingualism: Language and Cognition, in press.

[14] W. Francis, Cognitive integration of language and memory in bilinguals: semantic representation, Psychol. Bull. 125 (1999) 193222.

[15] W.J. Gehring, A.R. Willoughby, The medial frontal cortex and the rapid processing of monetary gains and losses, Science 295 (2002) 2279-2282. 
[16] M.S. George, T.A. Ketter, P.I. Parekh, N. Rosinsky, H. Ring, B.J. Casey, M.R. Trimble, B. Horowitz, P. Herscovitch, R.M. Post, Regional brain activity when selecting a response despite interference: an $\mathrm{H}_{2}^{15} \mathrm{O}$ PET study of the Stroop and emotional Stroop, Hum. Brain Mapp. 1 (1994) 194-209.

[17] M.O. Glaser, W.R. Glaser, Time course analysis of the Stroop phenomenon, J. Exp. Psychol.: Hum. Percept. Perform. 8 (1982) $875-894$.

[18] C.B. Holroyd, M.G.H. Coles, The neural basis of human error processing: reinforcement learning, dopamine, and the error-related negativity, Psychol. Rev., in press.

[19] P. Indefrey, W.J.M. Levelt, The neural correlates of language production, in: M. Gazzaniga (Ed.), The New Cognitive Neurosciences, MIT Press, Cambridge, MA, 2000, pp. 845-865.

[20] P. Indefrey, W.J.M. Levelt, The spatial and temporal signatures of word production components, Cognition, in press.

[21] W.J.M. Levelt, Speaking: From Intention to Articulation, MIT Press, Cambridge, MA, 1989.

[22] W.J.M. Levelt, A. Roelofs, A.S. Meyer, A theory of lexical access in speech production, Behav. Brain Sci. 22 (1999) 1-38.

[23] S.J. Lupker, P. Brown, L. Colombo, Strategic control in a naming task: changing routes or changing deadlines?, J. Exp. Psychol.: Learn. Mem. Cognit. 23 (1997) 570-590.

[24] A.W. MacDonald, J.D. Cohen, V.A. Stenger, C.S. Carter, Dissociating the role of dorsolateral prefrontal and anterior cingulate cortex in cognitive control, Science 288 (2000) 1835-1838.

[25] C.M. MacLeod, Half a century of research on the Stroop effect: an integrative review, Psychol. Bull. 109 (1991) 163-203.

[26] C.M. MacLeod, P.A. MacDonald, Interdimensional interference in the Stroop effect: uncovering the cognitive and neural anatomy of attention, Trends Cogn. Sci. 4 (2000) 383-391.

[27] B. Maess, A.D. Friederici, M. Damian, A.S. Meyer, W.J.M. Levelt, Semantic category interference in overt picture naming: sharpening current density localization by PCA, J. Cogn. Neurosci. 14 (2002) $455-462$.

[28] P.K. McGuire, D.A. Silbersweig, C.D. Frith, Functional neuroanatomy of verbal self-monitoring, Brain 119 (1996) 101-111.

[29] E.K. Miller, The prefrontal cortex and cognitive control, Nat. Rev. Neurosci. 1 (2000) 59-65.

[30] D.A. Norman, T. Shallice, Attention to action: willed and automatic control of behavior, in: R.J. Davidson, G.E. Schwarts, D. Shapiro (Eds.), Consciousness and Self-regulation: Advances in Research and Theory, Vol. 4, Plenum Press, New York, 1986, pp. 1-18.

[31] J.V. Pardo, P. Pardo, K.W. Janer, M.E. Raichle, The anterior cingulate cortex mediates processing selection in the Stroop attentional conflict paradigm, Proc. Natl. Acad. Sci. USA 87 (1990) 256-259.

[32] T. Paus, Primate anterior cingulate cortex: where motor control, drive and cognition interface, Nat. Rev. Neurosci. 2 (2001) 417424.
[33] T. Paus, D.W. Perry, R.J. Zatorre, K.J. Worsley, A.C. Evans, Modulation of cerebral blood flow in the human auditory cortex during speech: role of motor-to-sensory discharges, Eur. J. Neurosci. 8 (1996) 2236-2246.

[34] B.S. Peterson, M.J. Kane, G.M. Alexander, C. Lacadie, P. Skudlarski, H.-C. Leung, J. May, J.C. Gore, An event-related functional MRI study comparing interference effects in the Simon and Stroop tasks, Cogn. Brain Res. 13 (2002) 427-440.

[35] B.S. Peterson, P. Skudlarski, J.C. Gatenby, H. Zhang, A.W. Anderson, J.C. Gore, An fMRI study of Stroop word-color interference: evidence for cingulate subregions subserving multiple distributed attentional systems, Biol. Psychiatry 45 (1999) 1237-1258.

[36] M.I. Posner, M.E. Raichle, Images of Mind, Freeman, New York, 1994.

[37] C. Price, P. Indefrey, M. van Turennout, The neural architecture underlying the processing of written and spoken word forms, in: C.M. Brown, P. Hagoort (Eds.), The Neurocognition of Language, Oxford University Press, Oxford, 1999, pp. 212-240.

[38] A. Rodriguez-Fornells, M. Rotte, H.-J. Heinze, T. Nösselt, T. Münte, Brain potential and functional MRI evidence for how to handle two languages with one brain, Nature 415 (2002) 10261029.

[39] A. Roelofs, A spreading-activation theory of lemma retrieval in speaking, Cognition 42 (1992) 107-142.

[40] A. Roelofs, The WEAVER model of word-form encoding in speech production, Cognition 64 (1997) 249-284.

[41] A. Roelofs, Goal-referenced selection of verbal action: modeling attentional control in the Stroop task, Psychol. Rev., in press.

[42] P.H. Schiller, Developmental study of color-word interference, J. Exp. Psychol. 72 (1966) 105-108.

[43] J.R. Stroop, Studies of interference in serial verbal reactions, J. Exp. Psychol. 18 (1935) 643-662.

[44] D.T. Stuss, D. Floden, M.P. Alexander, B. Levine, D. Katz, Stroop performance in focal lesion patients: dissociation of processes and frontal lobe lesion location, Neuropsychologia 39 (2001) 771-786.

[45] D. Swick, J. Jovanovic, Anterior cingulate cortex and the Stroop task: neuropsychological evidence for topographic specificity, Neuropsychologia 40 (2002) 1240-1253.

[46] S.F. Taylor, S. Kornblum, E.J. Lauber, S. Minoshima, R.A. Koeppe, Isolation of specific interference processing in the Stroop task: PET activation studies, NeuroImage 6 (1997) 81-92.

[47] U. Turken, D. Swick, Response selection in the human anterior cingulate cortex, Nat. Neurosci. 2 (1999) 920-924.

[48] P. Vendrell, C. Junqué, J. Pujol, M.A. Jurado, J. Molet, J. Grafman, The role of prefrontal regions in the Stroop task, Neuropsychologia 33 (1995) 341-352. 